Natural Hazards and Earth System Sciences (2004) 4: 793-798

SRef-ID: 1684-9981/nhess/2004-4-793

European Geosciences Union

(C) 2004 Author(s). This work is licensed

under a Creative Commons License.

\title{
Interpretation of the microwave non-thermal radiation of the Moon during impact events
}

\author{
V. Grimalsky ${ }^{1}$, A. Berezhnoy ${ }^{2,3}$, A. Kotsarenko ${ }^{4}$, N. Makarets $^{5}$, S. Koshevaya ${ }^{6}$, and R. Pérez Enríquez ${ }^{4}$ \\ ${ }^{1}$ Instituto Nacional de Astrofisica, Optica y Electronica (INAOE), Puebla, Mexico \\ ${ }^{2}$ Advanced Research Institute for Science and Engineering, Waseda University, Tokyo, Japan \\ ${ }^{3}$ Now at: Sternberg Astronomical Institute, Moscow University, Moscow, Russia \\ ${ }^{4}$ Centro de Geociencias, Juriquilla, UNAM, Querétaro, Mexico \\ ${ }^{5}$ Kyiv National Shevchenko University, Faculty of Physics, Kyiv, Ukraine \\ ${ }^{6}$ Universidad Autonoma del Estado de Morelos (UAEM), CIICAp, Cuernavaca, Mexico
}

Received: 30 June 2004 - Revised: 23 November 2004 - Accepted: 24 November 2004 - Published: 30 November 2004

Part of Special Issue "Precursory phenomena, seismic hazard evaluation and seismo-tectonic electromagnetic effects"

\begin{abstract}
The results of recent observations of the nonthermal electromagnetic (EM) emission at wavelengths of $2.5 \mathrm{~cm}, 13 \mathrm{~cm}$, and $21 \mathrm{~cm}$ are summarized. After strong impacts of meteorites or spacecrafts (Lunar Prospector) with the Moon's surface, the radio emissions in various frequency ranges were recorded. The most distinctive phenomenon is the appearance of quasi-periodic oscillations with amplitudes of 3-10 K during several hours. The mechanism concerning the EM emission from a propagating crack within a piezoactive dielectric medium is considered. The impact may cause the global acoustic oscillations of the Moon. These oscillations lead to the crackening of the Moon's surface. The propagation of a crack within a piezoactive medium is accompanied by the excitation of an alternative current source. It is revealed that the source of the EM emission is the effective transient magnetization that appears in the case of a moving crack in piezoelectrics. The moving crack creates additional non-stationary local mechanical stresses around the apex of the crack, which generate the non-stationary electromagnetic field. For the cracks with a length of $0.1-1 \mu \mathrm{m}$, the maximum of the EM emission may be in the $1-10 \mathrm{GHz}$ range.
\end{abstract}

\section{Introduction}

The prediction of earthquakes is an important problem, which is still far from a final solution. One of the possible methods for recognition of precursors of earthquakes is the investigation of the electromagnetic (EM) radiation of seismic origin. The EM radiation before earthquakes has been recorded in the $\mathrm{kHz}$ and $\mathrm{MHz}$ frequency ranges (Gokhberg et al., 1995). Also, the EM emission was measured in laboratory rock fracture experiments (Gershenzon et al., 1986).

Correspondence to: V. Grimalsky

(vgrim@inaoep.mx)
There are several probable models considering how the mechanic stress can be transformed into the EM radiation:

1. formation of new micro cracks in the rock,

2. charges arising at the peaks of existing cracks formed under the action of an increasing load,

3. piezoelectric and piezomagnetic effects.

The source of the EM radiation is located near the epicenter of an earthquake with depths of about $10-30 \mathrm{~km}$. Thus, only the low frequency part of the total EM radiation can reach the Earth's surface, because of the finite conductivity of the Earth's crust, and in fact the total spectrum of the EM emission cannot be measured.

However, the non-thermal origin of the EM radiation after collisions of man-made and natural objects with the Moon has been measured. In this case, the source of the radiation is located directly on the Moon's surface, and there exists the possibility of recording the total spectrum of the EM emission. Therefore, the investigation of non-thermal emissions from the surface of the Moon may give some insights on the specific mechanisms of the EM radiation of seismic origin in the Earth.

\section{Review of radio observations of the Moon}

The main source of lunar radio emission at centimeter wavelengths is the thermal emission of a brightness temperature equal to $250 \mathrm{~K}$. The intensity of the thermal radio emission changes slowly with a period equal to one month. However, after strong impacts of meteorites or spacecrafts with the Moon's surface, the quick changes of the intensity of the lunar radio emission in various frequency ranges were detected. The collision between the American spacecraft Lunar 


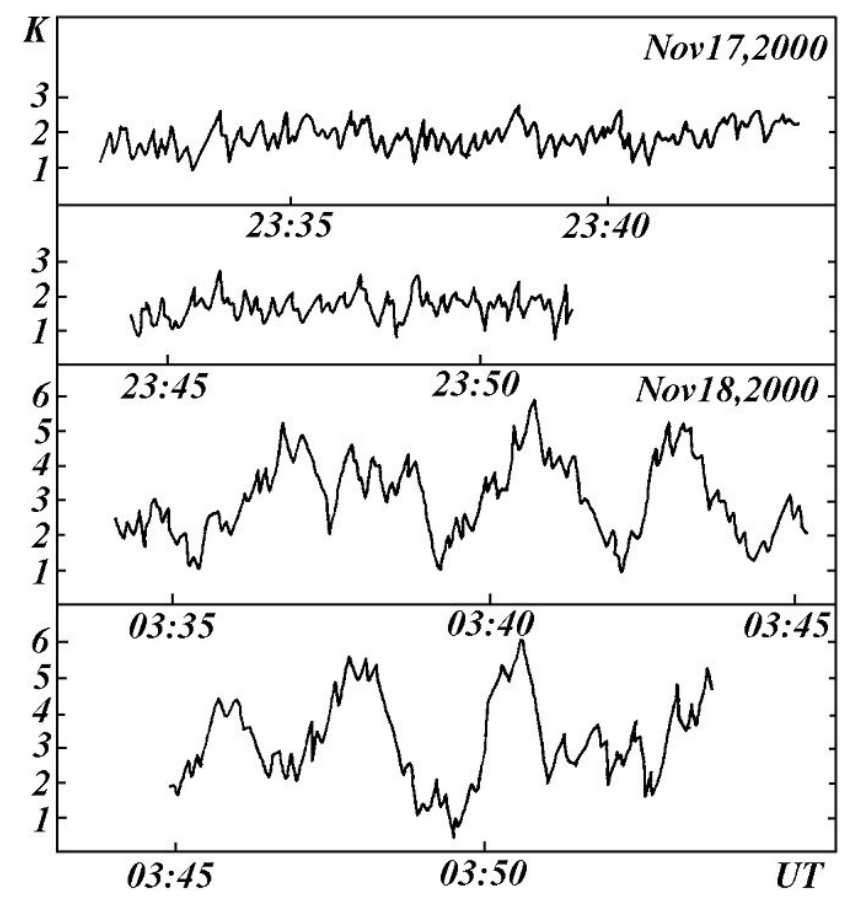

Fig. 1. Variations of the lunar radio flux at $2.46 \mathrm{~cm}$ on $17 / 18$ November 2000. Observations were conducted with the $32 \mathrm{~m}$ Ventspils radio telescope. The antenna temperature is given in degrees Kelvin.

Prospector and the Moon on 31 July 1999, was equivalent to a big moonquake, because the impact mass and velocity were $160 \mathrm{~kg}$ and $1.7 \mathrm{~km} / \mathrm{s}$, respectively. During the observations at $64 \mathrm{~m}$ Kalyazin Radio Telescope (Russia), variations of the lunar radio flux at wavelengths $\lambda_{0}=13$ and $\lambda_{0}=21 \mathrm{~cm}$ were detected on 30 July-2 August 1999 (Berezhnoy et al., 2000; Khavroshkin et al., 2001). There is a correlation between the variations of the received lunar signal at both wavelengths, giving evidence of a common mechanism of the radio emission (Berezhnoy et al., 2000). The average values of the amplitude of variations of the lunar radio brightness temperature were in the range $2-10 \mathrm{~K}$. Note that the impact of the Lunar Prospector was accompanied by the Perseid meteor stream, that could produce an additional prolonged source of EM emissions.

Some measurements of radio emissions under meteor showers on the Moon, using the $32 \mathrm{~m}$ radio telescope of the Ventspils International Radio Astronomy Center (Latvia) in 2000 and 2001, have been reported. The amplitude of fluctuations of the lunar radio flux at $\lambda_{0}=2.46 \mathrm{~cm}$ ( $44 \mathrm{MHz}$ bandwidth) increased significantly (3-5 times) during the Leonid 2000 meteor shower on 18 November 2000 (Berezhnoy et al., 2002). The sensitivity of the Ventspils radio telescope is $0.07 \mathrm{~K}$ and the measured tolerance ( $5 \sigma$ level) is $0.35 \mathrm{~K}$ at $1 \mathrm{~s}$ output time constant (Bervalds et al., 2001). The Leonid meteoroids can impact the Moon at relative velocities of up to $72 \mathrm{~km} / \mathrm{s}$; the mass of the biggest colliding meteoroids is of about 1-10 kg (Ortiz et al., 2002). The high kinetic energy collisions can excite seismic waves in the lunar body.
In the morning of 17 November 2000, the changes of the received lunar radio signal gradually continued for $5 \mathrm{~h}$ (see Fig. 1). There were at least two isolated events with valleyto-peak amplitudes of the oscillations of the antenna temperature exceeding $10 \mathrm{~K}$. On 18 November 2000, the valley-topeak amplitudes of the oscillations reached $10 \mathrm{~K}$. Most of the quasi-periodic oscillations had periods from 1 to $6 \mathrm{~min}$. Observed valley-to-peak oscillation amplitudes in November 2001, were larger than that in November 2000. The valley-topeak amplitudes of quasi-periodic oscillations reached $25 \mathrm{~K}$ on 18 and 19 November 2001. The most frequent periods found were $2.5,3.0,3.7$, and $6.0 \mathrm{~min}$. Those periods were similar to the periods detected during the Leonid 2000 meteor shower. The times of maxima of the amplitudes of the variations of the lunar radio flux agree well with predicted times of maxima of the Leonid activity on the Moon. This may be evidence that the amplitude of the variations of the lunar radio flux increases with the increasing Leonid activity on the Moon, if the instrumental effects are negligible.

A study of fluctuations of the lunar radio flux under the Lyrid shower was carried out at $1.3 \mathrm{~cm}, 6 \mathrm{~cm}$, and $18 \mathrm{~cm}$ on 16-20 April 2001 (Berezhnoy et al., 2002). The bandwidth was $\Delta f=150 \mathrm{MHz}$ at $1.3 \mathrm{~cm}$ and $\Delta f=6 \mathrm{MHz}$ at $6 \mathrm{~cm}$ and $18 \mathrm{~cm}$. An important feature is the periodic variations of the lunar radio flux. Periods from 2 to $15 \mathrm{~min}$ were similar to those at other wavelengths. The amplitude of the variations of lunar flux was about $1-10 \mathrm{~K}$ for all wavelengths.

All these observations of the Moon were conducted with the same antenna. This means that the detected variations of the lunar flux could be caused by either atmospheric turbulence or an instability of the receiver. In order to study the possible role of instrumental effects, simultaneous observations of the center of the Moon at $6.2 \mathrm{~cm}$ were conducted at Pushchino radio telescope (Russia) and Simeiz radio telescope (Ukraine) during 17-19 November 2001. These observations are important because they were conducted simultaneously at similar frequencies as the Ventspils observations. The frequency and the bandwidth of the receiver at Simeiz are $4.866 \mathrm{GHz}$ and $2 \mathrm{MHz}$, respectively. The frequency and bandwidth of the receiver at Pushchino are $4.830 \mathrm{GHz}$ and $6 \mathrm{MHz}$, respectively. The diameter of the antennas of both radio telescopes is $22 \mathrm{~m}$. No correlation between fluctuations of the lunar radio flux at $4.866 \mathrm{GHz}$ and $4.83 \mathrm{GHz}$ was found. Thus, the detected periodicities of the lunar flux at other wavelengths should be checked again by simultaneous observations at two isolated antennas. This means that the intensity of the lunar radio emission of seismic origin is less than the sensitivity of both telescopes $(3 \mathrm{~K}$ or $5000 \mathrm{Jy}$ at $6.2 \mathrm{~cm})$. We cannot estimate the upper level of the intensity of the lunar radio emission of seismic origin at $2.46 \mathrm{~cm}$, because the lunar radio flux was not calibrated at these observations. Radio flashes caused by collisions of $\mathrm{kg}$ meteoroids with the Moon were not detected in the telescopes (Volvach et al., 2004).

Notice that the absolute values of the lunar radio brightness temperature were not measured during these observations. The calibration of the lunar radio flux was conducted 
every $30-60 \mathrm{~min}$. For this reason, only the information concerning the temporal variability of the lunar radio flux at $1 \mathrm{~s}$ time resolution was obtained. The values of the observed periods of the lunar radio flux at $2.46 \mathrm{~cm}$ and $6.2 \mathrm{~cm}$ during the Leonid meteor shower in 2001 were different. This means that the excitations of free oscillations of the Moon cannot explain the periodicities of the lunar radio flux. The Leonid meteor shower activity shows the periodicities in the minute range (Singer et al., 2000). Probably, the periodicities of the Leonid meteor shower activity may be responsible for the appearance of periodicities in the lunar radio flux.

\section{Piezoelectric mechanism of electromagnetic emission}

In this section we will propose a mechanism explaining the origin of the microwave lunar radio emission. The absolute values of intensity of the radio emission produced by this mechanism can also be estimated. The observed EM emission can be explained by various mechanisms, such as the motion of charged dislocations, piezoeffect, and plasma emission. In this work, the mechanism concerning the EM emission from a propagating crack within a piezoactive dielectric medium is considered. The impact can cause global acoustic oscillations of the Moon. These oscillations can lead to cleaving mechanically weak rocks on the whole Moon's body, and the shock necessarily leads to the cracking of a local part of its surface. The piezoelectric $\mathrm{SiO}_{2}$ is a constituent of the lunar minerals; therefore, it can radiate after cracking.

We are considering a piezoelectric plate to which a mechanical stress of a value $\boldsymbol{P}_{0}$ is applied along the OY-axis and normal to the optical OZ-axis, as shown in Fig. 2. For our estimations, all sizes of the plate $(L, H, h)$ are assumed to be equal and we take quartz $\left(\mathrm{SiO}_{2}\right)$ as a piezoelectric material. The piezoeffect produces an electric field along the piezoaxes OX and OY. It is important that the fracturing of crystals, known as cleavage (Shuvalov et al., 1981), can occur in distinctive crystallographic planes. According to this, the crack caused by $\boldsymbol{P}_{0}$ can expand along the axis OX, as shown in the Fig. 2. When the crack exists inside the plate, it begins to move through the crystal only if the applied mechanic stress exceeds certain value. The velocity of the crack in quartz is not greater than the velocity of the transverse acoustic waves. It is necessary to analyze the nonstationary problem because of the following reasons. First of all, an EM emission could exist as a result of the relaxation of the electron system, on the created surfaces in any materials. However, the time of this relaxation is very short and the frequency of the corresponding EM is very high. Also, the motion of the unloading wave from the crack occurs, and non-stationary local mechanical stresses around the crack in the piezoelectric appear.

The equations of motion are obtained by using a free en$\operatorname{ergy} \Psi$ of a crystal. The free energy $\Psi=\Psi\left(u_{k l}, T, E_{k}, B_{k}\right.$, $\left.\alpha_{1}, \ldots, \alpha_{N}\right)$ is a function of the deformation tensor $u_{k l}$, temperature $T$, external electric and magnetic fields $\boldsymbol{E}, \boldsymbol{B}$, and, perhaps, of some $N$ internal parameters $\alpha_{k}$. If a dielectric is

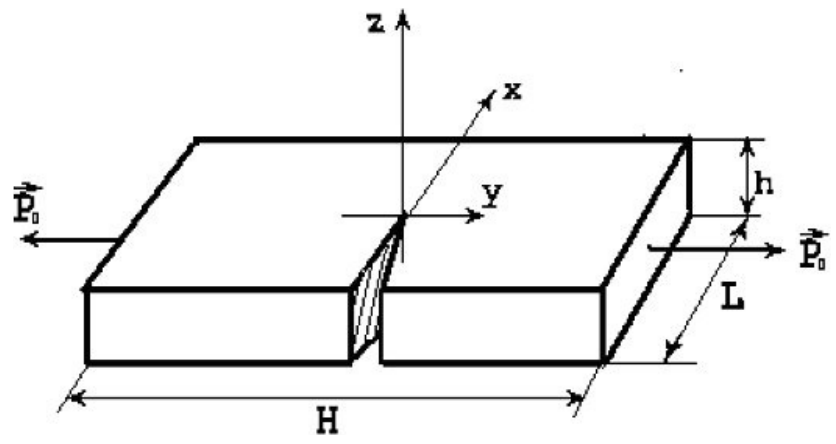

Fig. 2. Model of a moving crack.

under the influence of small deformations and small electric fields, the equations of motion can be derived in the linear theory approach. This assumption gives us the possibility to analyze the emission phenomenon in a linear approach. We use the Maxwell's equations for the description of electromagnetic waves

$\operatorname{rot} \boldsymbol{H}=\frac{1}{c} \frac{\partial \boldsymbol{D}}{\partial t}, \operatorname{div} \boldsymbol{H}=0$,
$\operatorname{rot} \boldsymbol{E}=-\frac{1}{c} \frac{\partial \boldsymbol{H}}{\partial t}, \operatorname{div} \boldsymbol{D}=0$,

and the equations of motion for a piezoelectric system

$\rho \frac{\partial^{2} U_{i}}{\partial t^{2}}=\frac{\partial \sigma_{i j}}{\partial x_{j}}$,

$\sigma_{i j}=c_{i j k l} U_{k l}-e_{k i j} E_{k}, D_{i}=\varepsilon_{i j} E_{j}+e_{i j k} U_{j k}$,

where $\boldsymbol{D}$ is the electric induction, $c$ is the velocity of light, $\sigma_{i j}, U_{i}$ are the components of stresses and mechanic displacement, respectively; $c_{i j k l}, e_{i j k}, \varepsilon_{i j}$ are the components of the tensors of the elastic constants, the piezoelectric constants, and the dielectric permittivity, respectively (Makarets et al., 2002).

Substituting $\boldsymbol{D}$ from the Eqs. (2) into the Maxwell's equations and taking into account the definition of the linear deformation tensor $U_{k l}=(1 / 2)\left(\partial U_{k} / \partial x_{l}+\partial U_{l} / \partial x_{k}\right)$, one can obtain the wave equation for the components of the electric field $E_{i}(\boldsymbol{r}, t)$, which can be presented as:

$$
\begin{aligned}
& \Delta E_{i}-\frac{\partial}{\partial x_{i}} \operatorname{div} \boldsymbol{E}-\frac{1}{c^{2}} \frac{\partial^{2}}{\partial t^{2}}\left(\varepsilon_{i j} E_{j}\right)= \\
& \frac{1}{2 c^{2}} e_{i k l}\left(\frac{\partial}{\partial x_{l}} \frac{\partial^{2} U_{k}}{\partial t^{2}}+\frac{\partial}{\partial x_{k}} \frac{\partial^{2} \boldsymbol{U}_{l}}{\partial t^{2}}\right) \equiv \boldsymbol{S}_{i}(\boldsymbol{r}, t) .
\end{aligned}
$$

The right hand side $\boldsymbol{S}(\boldsymbol{r}, t)$ of the Eq. (3) is the source of the EM emission, and it is connected with the mechanical displacement vector $\boldsymbol{U}$. As our calculations have shown (Makarets et al., 2002), this source is equivalent to the effective transient magnetization vector under the above mentioned conditions.

It is possible to analyze three basic cases of fracturing (see Fig. 3), which determine three configurations of the mechanical displacement vector $\boldsymbol{U}$. In the static case, if the crack 


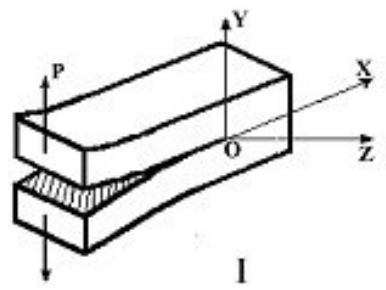

I

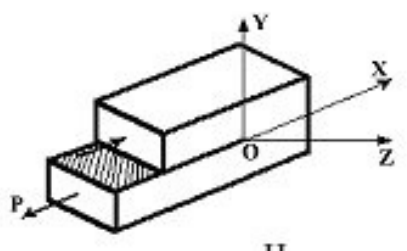

II

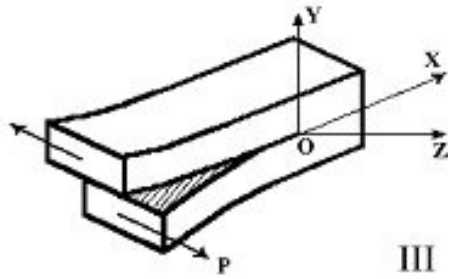

Fig. 3. Model of various types of cracks.

does not move, the vector $\boldsymbol{U}$ is determined only by the coordinates, and the right hand side of Eq. (3) is zero. Hence the $\mathrm{EM}$ emission is absent. If the crack moves, the displacement vector $\boldsymbol{U}$ depends on the time $t$ and the spatial coordinates $\boldsymbol{r}$, and $\boldsymbol{S}(\boldsymbol{r}, t) \neq 0$. The problem for a calculation of the EM emission caused by a moving crack in the quartz has been solved in the first approach by Makarets et al. (2002), and the details of the calculation can be found there. The most appropriate distribution of the elastic deformation for the uniformly moving crack, used in our calculations, is given by Gus' and Zozulya (1993). It has been used to obtain the right hand side of the Eq. (3). In this solution, the influence of the crystal symmetry and the electric field on the values of $\boldsymbol{U}(\boldsymbol{r}, t)$ and, hence, $\boldsymbol{S}(\boldsymbol{r}, t)$ is not taken into account. Therefore, the results of Makarets et al. (2002) are the zeroth approximation.

First of all, we discuss the source of electromagnetic waves. We have shown (Makarets et al., 2002) that Eq. (3) can be transformed into a classical wave equation (Landau and Lifshitz, 1960) which has the source term $\left(4 \pi / c^{2} \operatorname{rot}(\partial \boldsymbol{M}(r, t) / \partial t)\right.$ in the right hand side. Therefore, an effective magnetization current $\boldsymbol{j}=\operatorname{rot} \boldsymbol{M}(\boldsymbol{r}, t)$ exists in the piezoelectric quartz crystal around the moving crack, and a variable magnetization vector $\boldsymbol{M}(\boldsymbol{r}, t)=\boldsymbol{m}(\boldsymbol{r}, t)+\boldsymbol{m}_{0}(\boldsymbol{r})$ appears as the source of the accompanying EM emission. It is possible to calculate the vector $\boldsymbol{m}(\boldsymbol{r}, t)$, and the vector $\boldsymbol{m}_{0}(\boldsymbol{r})$ is a constant, which can be omitted. It is important that the polarization current in the dielectric quartz is of a vortical character and creates an effective magnetic moment, i.e. it is analogous to an effective magnetization current. Our investigations have demonstrated that the propagation of the crack with the front parallel to the crystalline optical axis can create a similar effective magnetic moment in the crystals, which belong to the symmetry groups 422 and 622 for the cracks of the kind III and to the $\overline{6}$ and $\overline{6} \mathrm{~m} 2$ symmetry groups for the cracks of the kinds I and II (see Fig. 3).

Let us assume that a quartz crystal includes an initial crack of a length close to the interatomic distance $l_{0} \approx 0.1 \mathrm{~nm}$. Under mechanical stresses, the crack moves through the crystal with a velocity $V \approx 2 \mathrm{~km} / \mathrm{s}$ during a time $T_{0} \approx L / V$, where $L$ is the final length of the crack. Under a motion of the surface acoustic wave along the Moon's surface, cracks of various lengths $L$ can be produced. Those cracks are the sources of effective alternative magnetic moments, which are oriented along the axis OZ. The micro cracks $(L \sim 1 \mu \mathrm{m})$ can be considered as possible sources of the microwave radiation.

\section{Frequency dependence of the electromagnetic emis- sion}

The dependence of the intensity of the radio emission of a seismic origin versus frequency was measured before strong earthquakes in Greece (Eftaxias et al., 2003). Based on observations in the $\mathrm{kHz}$ and $\mathrm{MHz}$ frequency ranges, it was established that the intensity of the radio emission of seismic origin increased as the square of the wavelength. This dependence may be not so strong in the case of meteoroid impacts on the Moon due to the absence of the skin effect. Unfortunately, based on the performed radio observations of the Moon, it is impossible to estimate the frequency of the maximum intensity of the lunar radio emission of seismic origin. In Makarets et al. (2002) it was shown that the calculated intensity of the EM emission from the cracks possesses an infinite number of periodically located maxima, and its first maximum value appears at the frequency $\omega_{\max } \approx \pi(V / L)$, and the corresponding wavelength is $\lambda_{\max } \approx 2 L(c / V)\left(\varepsilon_{11}\right)^{-1 / 2}$. The next maxima of the intensity of the EM radiation correspond to shorter waves, but they have no physical sense. An explanation is as follows. In simulations (Makarets et al., 2002), it was assumed that the velocity $V$ of the moving crack is constant: $V=V_{0}$ during a time $T=L / V_{0}$. Therefore, at the beginning of the motion and at its end, the acceleration is infinite. A more realistic model for the frequency dependence of the intensity of the EM radiation should be applied. Namely, the acceleration of the crack can be approximated by a part of a sinusoid:

$$
\begin{aligned}
& \frac{d V}{d t}=a_{0} \sin \left(\omega_{0} t\right), \quad 0<t<T ; \quad \omega_{0}=2 \pi / T ; \\
& V_{\max }=V_{0} ; \quad T=2 V_{0} / L .
\end{aligned}
$$

or step-like:

$\frac{d V}{d t}= \begin{cases}a_{0}, & 0<t<T / 2 \\ -a_{0}, & T / 2<t<T .\end{cases}$

A comparison of the dependencies of the intensity of the EM radiation on frequency for various cases of approximation of the velocity of the moving crack is presented in Fig. 4. One can see that the curves near the first (real) maximum are the same for all kinds of approximations. The corresponding value of the frequency is $\omega_{\max } \approx \omega_{0} \approx \pi V_{0} / L$. The nonsingular approximations also give the same result for the frequency dependence at higher frequencies. If an approximation of the 


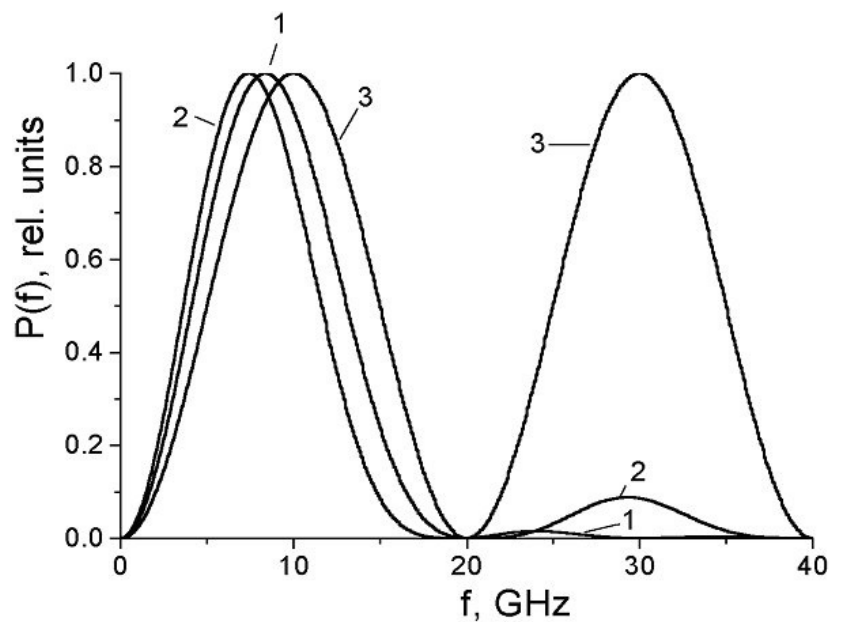

Fig. 4. Frequency dependencies of the EM intensity emitted in the direction $\theta=\psi=\pi / 2$ by a crack of the type III for various accelerations of the crack. 1 is for sine-like approximation (Eq. 4), 2 is for step-like approximation (Eq. 5), 3 is for the approximation with constant velocity (singular acceleration). The final length of the moving crack is $L=0.1 \mu \mathrm{m}$.

velocity of the moving crack is discontinuous, then the nonphysical higher maxima possess the same value as the first true maximum. Therefore, we need to use here the smooth sine-like approximation for the velocity (Eq. 4). The value of the maximum velocity $V_{0}$ is $V_{0}=2 \mathrm{~km} / \mathrm{s}$.

The frequency dependencies of the intensity of the EM radiation for the single micro cracks of different lengths are presented in Fig. 5. They are plotted for the following conditions: $i$ ) the direction of the EM emission is $\theta=\psi=\pi / 2$, $i i)$ the crack-type is III. The angular distribution of the radiation possesses a sharp orientation in the piezoactive plane $\theta=\pi / 2$. It should be noted that under real conditions the amplitudes of the maximum are sensitive to the crystal shape and, therefore, some directions of the maximum EM radiation can be changed. One can see that under the motion of micro cracks the maximum of the EM radiation is in the microwave range.

It should be noted that the low frequency EM emissions can be registered during volcano eruptions, when a fracture is located at the surface of the volcano. In the case of a deep fracture, the EM waves at higher frequencies dissipate, and only ELF (extremely low frequency) and VLF (very low frequency) waves are registered at the Earth's surface. Hence, it is necessary to use very sensitive sensors to register the higher frequencies, because the high frequency EM waves attenuate very quickly with distance in the lithosphere.

\section{Conclusions}

The frequency range of the EM radiation due to crackening in piezorocks, which may be recorded experimentally, depends on the depth of the localization of the source. If the source is located within the depth of the planetary crust, the higher

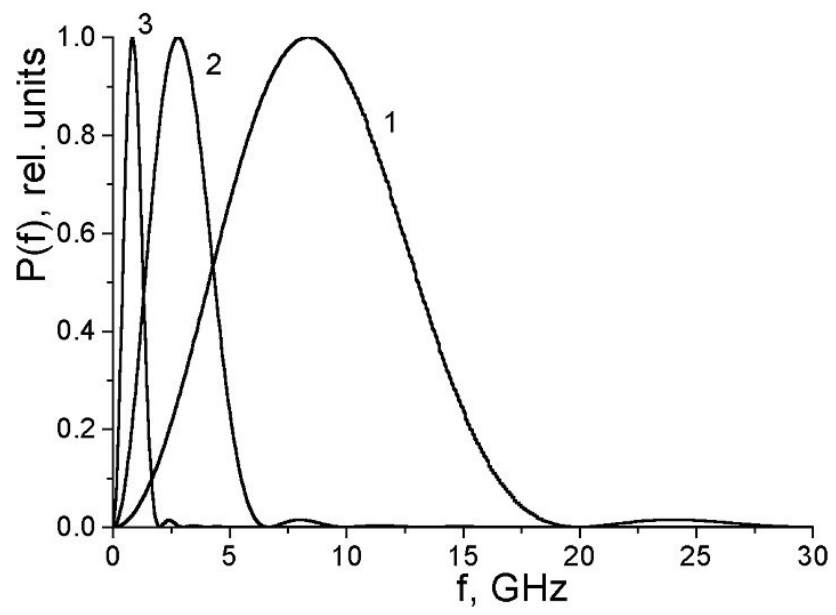

Fig. 5. Frequency dependencies of EM intensity for non-singular dependence of the velocity of the crack on time for cracks with different lengths: 1 is for the length of the crack $L=0.1 \mu \mathrm{m}, 2$ is for $L=0.3 \mu \mathrm{m}, 3$ is for $L=1 \mu \mathrm{m}$.

frequency components are suppressed during their propagation, due to the skin effect. However, when the source is located at the surface of a planet, all the spectral components of EM radiation may be measured. Therefore, the possible origin of non-thermal emission from the Moon's surface may be explained by the EM emission under micro fracturing of piezorocks during the propagation of the acoustic surface waves. For the cracks of lengths $L=0.1 \mu \mathrm{m}-10 \mu \mathrm{m}$, the maximum intensity of the EM emission takes place at $\sim 0.1-10 \mathrm{GHz}$. But the tail of the emission spreads up to $10-$ $30 \mathrm{GHz}$.

Acknowledgements. A. A. Berezhnoy is supported by a postdoctoral fellowship grant (No. P02059) from the Japanese Society for the Promotion of Science (JSPS). The authors are grateful to J. De la Hidalga-W. for discussions of the results and the correction of the text of the paper, as well as to the referees C.-V. Meister and O. A. Pokhotelov for stimulating remarks that have given us a possibility to improve the text of the manuscript.

Edited by: P. F. Biagi

Reviewed by: C.-V. Meister and O. A. Pokhotelov

\section{References}

Berezhnoi, A. A., Gusev, S. G., Khavroshkin, O. B., Poperechenko, B. A., Shevchenko, V. V., and Tzyplakov, V. A.: Radio Emission of the Moon before and after the Lunar Prospector impact, Proc. of the 4th Internat. Conference on Exploration and Utilization of the Moon, 179-181, 2000.

Berezhnoy, A. A., Bervalds, E., Khavroshkin, O. B., Kovalenko, A. V., Ozolins, G., Paupere, M., Smirnov, G. T., and Tsyplakov, V. V.: Radio observations of the Moon during the activity periods of Leonid and Lyrid meteor streams, Baltic Astronomy, 11, 507527, 2002.

Bervalds, E., Ozolinsh, G., Paupere, M., Rakitko, G., Bezrukov, D., Bezrukov, V., Bondarenko, V., and Sika, Z.: Observation of 
lunar radio emission during the year 2000 Leonid meteor shower maximum, VIRAC scientific preprint, 1, 5, 2001.

Eftaxias, K., Kapiris, P., Polygiannakis, J., Peratzakis, A., Kopanas, J., Antonopoulos, G., and Rigas, D.: Experience of short term earthquake precursors with VLF-VHF electromagnetic emissions, Natural Hazards and Earth System Sciences, 3, 217-228, 2003,

SRef-ID: 1684-9981/nhess/2003-3-217.

Gershenzon, N. I., Zilpimiani, D. O., Mandzhgaladze, P. V., and Pokhotelov, O. A.: Electromagnetic radiation emitted by the apex of a crack during failure in LiF crystals, Doklady AN SSSR (Sov. Phys. - Doklady), 228, 75-78, 1986.

Gokhberg, M. B., Morgounov, V. A., and Pokhotelov, O. A.: Earthquake prediction - Seismoelectromagnetic phenomena, Amsterdam, Gordon and Breach Publishers, 193, 1995.

Gus', A. N. and Zozulya, V. V.: Brittle fracture of materials by dynamical loadings. In: Non-classical problems of the fracture mechanics, edited by Gus', A. N., Kiev, Naukova dumka, 4, 2, 240, 1993.

Khavroshkin, O. B., Tsyplakov, V. V., Poperechenko, B. A., Berezhnoi, A. A., and Shevchenko, V. V.: Modification of the Moon's microwave radiation by the fall of Lunar Prospector, Doklady Earth Sciences, 376, 1, 90-92, 2001.
Landau, L. and Lifshitz, E. Electrodynamics of Continuous Media, Pergamon, London, 480, 1960.

Makarets, M., Koshevaya, S., and Gernets, A.: EM Radiation Caused by the Fracturing of Piezoelectrics in Rocks, Physica Scripta, 65, 3, 268-272, 2002.

Ortiz, J. L., Quesada, J. A., Aceituno, J., Aceituno, F. J., and Bellot Rubio, L. R.: Observation and interpretation of Leonid impact flashes on the Moon in 2001, Astrophys. J., 576, 1, 567-573, 2002.

Shuvalov, L. A., Urusovskaya, A. A., Zholudev, I. S. et al.: Physical properties of crystals, in Modern crystallography, edited by Vainshtein, B. K., Moscow, Nauka, 4, 496, 1981.

Singer, W., Molau, S., Rendtel, J., Asher, D. J., Mitchell, N. J., and von Zahn, U.: The 1999 Leonid meteor storm: verification of rapid activity variations by observations at three sites, Monthly Notices of the Royal Astronomical Society, 318, 3, L25-L29, 2000.

Volvach, A. E., Berezhnoy, A. A., Khavroshkin, O. B., Kovalenko, A. V., and Smirnov, G. T.: The simultaneous observations of the Moon at $6.2 \mathrm{~cm}$ using $22 \mathrm{~m}$ radio telescopes at Pushchino and Simeiz during Leonid meteor shower on November 2001, Kinematics and Physics of Celestial Bodies, 22, 32-36, 2004. 